Proyectos empresariales industriales

Industrial business projects 


\title{
Estudio para la instalación de una planta productora de mazamorra de tocosh con maca, quinua y leche
}

\author{
Hugo Lechuga Gilt \\ Universidad de Lima. Lima, Perú \\ Correo electrónico: hugolech@outlook.com \\ Hugo Ilan Salas Ramírez \\ Universidad de Lima. Lima, Perú \\ Correo electrónico: hugo_ila_3@hotmail.com \\ Recibido: 19/4/2013 / Aprobado: 28/5/2013
}

Resumen: El tocosh es un producto andino hecho a partir de papa nativa, la cual pasa por un proceso de fermentación durante 6 meses, luego de este lapso se obtiene el tocosh, que es consumido en forma tradicional como mazamorra. Dado el creciente interés del mercado peruano por revalorar los productos andinos, el presente estudio busca establecer la viabilidad de mercado, tecnológica y económica de la producción y comercialización de una mazamorra andina hecha a base de tocosh y enriquecida con otros ingredientes andinos como la maca y la quinua, además de la leche.

Palabras clave: tocosh / papas fermentadas / mazamorras / postres alternativos / quinua / maca

\section{Project to install a producing plant of pudding made of tocosh, maca, quinoa and milk}

ABstract: The tocosh is an andean product made from native potatoes which go through a fermentation process of six months, after this period the tocosh is obtained and it is consumed in a traditional way as pudding. Due to the increasing interest of the Peruvian market to revaluate its andean products, this project aims to establish the technological and economic viability of the production and commercialization of an andean pudding made of tocosh, enriched with other andean ingredients such as maca, quinoa and milk.

Keywords: tocosh / fermented potatoes / pudding / alternatives desserts / quinua / maca 


\section{INTRODUCCIÓN}

Uno de los postres más populares en Latinoamérica es la mazamorra. $\mathrm{Su}$ origen proviene de varias tradiciones precolombinas, es preparada de diferentes formas y con distintos ingredientes en cada uno de los países en el que se consume. En el Perú la tradición de consumir mazamorra se remonta a la época la Colonia; la presentación más popular es la mazamorra morada, que sigue siendo la más consumida.

Sin embargo, aunque el consumo de mazamorra creció en el país durante la época colonial, anteriormente se consumían diversos tipos de este producto, a los que se conocía con el nombre de apis, que era preparado con distintos ingredientes propios de las zonas altoandinas. Una de estas mazamorras tradicionales y milenarias es la mazamorra de tocosh, la cual es consumida en grandes cantidades por los pobladores de la sierra de Huánuco y Ancash, aunque últimamente su consumo se ha extendido hasta la ciudad de Lima, donde es buscada por aquellos que ya la han probado y saben de su poder nutritivo y medicinal.

El fin del presente proyecto es proponer un producto que ponga al alcance de todas las personas una mazamorra andina que tenga como principal ingrediente el tocosh, en una presentación en polvo lista para ser preparada de manera fácil y sencilla, fortalecida con otros ingredientes andinos como la maca y la quinua, además de leche en polvo y fécula de papa.

El estudio detalla el mercado en el cual se quiere introducir el producto, la localización de la planta productora de mazamorra, los aspectos de ingeniería que serán necesarios para el buen funcionamiento de la planta propuesta, los costos y las inversiones implicadas con la puesta en marcha del proyecto, analizados desde el punto de vista económico y financiero, donde se determina su rentabilidad. Finalmente se trata el impacto social que conlleva su realización.

\section{ESTUDIO DE MERCADO}

\subsection{Usos y características del producto}

El producto es una mezcla de harinas de tocosh, maca y quinua, además de leche en polvo, azúcar y fécula de papa mezclados en proporciones específicas para su preparación inmediata en forma de mazamorra. Se caracteriza especialmente por ser novedoso y utilizar materia prima propia de los Andes peruanos. 


\subsection{Selección del mercado meta}

El mercado meta está conformado por los consumidores de los niveles socioeconómicos $\mathrm{B}$, C y D de Lima metropolitana, sin distinción de edades, por tratarse de un producto de consumo familiar. La población total de nuestro mercado seleccionado será de 6.259.526 personas.

\section{3 Área de estudio}

Dado que Lima es la ciudad que concentra la mayor cantidad de población en el país, representa un mercado muy atractivo para un producto con características de consumo masivo; además, existe la tradición de que a sus habitantes les gusta mucho la mazamorra, de ahí que a los oriundos de la capital se les llame «limeños mazamorreros»; por ello, el estudio de mercado para el presente proyecto se realizó en Lima metropolitana, específicamente en los distritos que representan los mayores porcentajes de habitantes correspondientes a los niveles socioeconómicos $\mathrm{B}, \mathrm{C}$ y D, a los que irá dirigido el producto propuesto debido a que gran cantidad de emigrantes se encuentran concentrados en estos niveles, lo mismo que el mayor volumen poblacional de la urbe (APEIM, 2011).

Se realizaron consultas a fuentes secundarias para obtener datos históricos de la demanda del producto, con dicha información se obtuvo la siguiente demanda interna aparente:

Tabla 1

Demanda interna aparente (DIA) (toneladas)

\begin{tabular}{ccccc}
\hline Año & Producción & Importaciones & Exportaciones & DIA \\
\hline 2007 & $3.175,82$ & 318,96 & 38,64 & $3.456,14$ \\
2008 & $3.408,11$ & 143,51 & 44,64 & $3.506,99$ \\
2009 & $4.088,91$ & 112,71 & 28,34 & $4.173,28$ \\
2010 & $4.422,50$ & 9,82 & 44,67 & $4.387,65$ \\
2011 & $4.586,82$ & 0,00 & 53,89 & $4.532,93$ \\
\hline
\end{tabular}

Fuentes: Datatrade (2012) y Alicorp (2011, estadística interna).

Elaboración propia. 
Para el desarrollo de la demanda potencial se realizó una encuesta con muestreo no probabilístico hecha por conveniencia en los mercados Unicachi, en Comas; Ceres, en Ate; y Magdalena, en Magdalena del Mar.

Según los resultados obtenidos de la encuesta se halló que cada ama de casa prepara en promedio 22 dulceras de mazamorra de 100 gramos cada una al mes. Si se tiene en cuenta que la población total de amas de casa es de 1.791.000 personas, se obtiene que el requerimiento mensual de mazamorra preparada es de 3.952 toneladas al mes.

Considerando la relación de mazamorra en polvo y mazamorra preparada, se pudo hallar que la demanda potencial total anual de mazamorra en polvo es de 6.776 toneladas. Para proyectar la demanda se consideró la población actual de amas de casa dentro del grupo seleccionado (1.791.000 personas), el consumo de cada una de ellas de mazamorras en general y la tasa de crecimiento poblacional de Lima, que es de 1,94\% anual (INEI, 2011).

Tabla 2

Evolución de la demanda potencial (toneladas)

\begin{tabular}{lll}
\hline Año & Población & Cantidad \\
\hline 2012 & 1.791 .000 & $6.776,52$ \\
2013 & 1.825 .745 & $6.907,98$ \\
2014 & 1.861 .165 & $7.041,99$ \\
2015 & 1.897 .271 & $7.178,61$ \\
2016 & 1.934 .079 & $7.317,87$ \\
2017 & 1.971 .600 & $7.459,84$ \\
2018 & 2.009 .849 & $7.604,56$ \\
2019 & 2.048 .840 & $7.752,09$ \\
2020 & 2.088 .587 & $7.902,48$ \\
2021 & 2.129 .106 & $8.055,79$ \\
\hline
\end{tabular}

Elaboración propia.

Teniendo en consideración el mismo ratio de crecimiento poblacional de 1,94\% anual se realizó la proyección de la evolución de la demanda interna aparente para los próximos 10 años, que se muestra a continuación. 
Tabla 3

Proyección DIA

(toneladas)

\begin{tabular}{cc}
\hline Año & Cantidad \\
\hline 2012 & $4.620,87$ \\
2013 & $4.710,51$ \\
2014 & $4.801,90$ \\
2015 & $4.895,05$ \\
2016 & $4.990,02$ \\
2017 & $5.086,82$ \\
2018 & $5.185,51$ \\
2019 & $5.286,11$ \\
2020 & $5.388,66$ \\
\hline 2021 & $5.493,20$ \\
\hline
\end{tabular}

Elaboración propia.

La mayor cantidad de personas de la ciudad de Lima se ubican en estos estratos socioeconómicos, aunque residen en distintas zonas de la ciudad. Los distritos que presentan mayor presencia de dichos NSE son San Miguel, Jesús María, Lince, Magdalena, Pueblo Libre, Rímac, La Victoria, Los Olivos, San Martín de Porres, Independencia, San Juan de Lurigancho, Santa Anita, Ate, Surquillo, Chorrillos, San Juan de Miraflores, Puente Piedra, Comas, Lurín, Carabayllo y Pachacamac (APEIM, 2011).

El producto propuesto está orientado al público de dos maneras: como un postre alternativo con propiedades favorables y como una forma más cómoda de consumir tocosh para quienes lo conocen y suelen consumirlo.

\subsection{Determinación de la demanda para el proyecto}

La demanda para el proyecto se determinó a partir de la información obtenida en la encuesta y los datos analizados de la oferta y la demanda actuales. Como ya se mostró anteriormente, a partir de los datos primarios recogidos se pudo dimensionar y proyectar la demanda potencial que tienen las mazamorras en polvo para el público objetivo, 
y a partir de los datos secundarios históricos consultados se construyó la demanda interna aparente actual, la cual también se proyectó, encontrándose una diferencia entre ambos tipos de datos, con lo cual se concluyó que existe una demanda no cubierta para los próximos años, parte de la cual se pretende cubrir con la mazamorra que se propone. Estos datos obtenidos fueron multiplicados por el factor de intención de compra ajustado por la intensidad de compra; datos que fueron hallados con los resultados de la encuesta, encontrándose que hay una intención de compra de $88 \%$ y una intensidad de compra de $57,27 \%$, de lo que resulta un porcentaje de intención de compra ajustado de 50,4\%.

Debido a que nuestro producto es innovador y busca insertarse en un mercado muy competitivo, donde participan empresas muy grandes especialistas en el rubro alimenticio, es prudente considerar que solo un $12 \%$ de la población identificada como demanda podría ser consumidor constante del producto propuesto.

Tabla 4

Demanda para el proyecto (toneladas)

\begin{tabular}{ccc}
\hline Año & $\begin{array}{c}\text { Demanda } \\
\text { no cubierta }\end{array}$ & $\begin{array}{c}\text { Demanda para el } \\
\text { proyecto }\end{array}$ \\
\hline 2012 & $1.086,45$ & 130,37 \\
2013 & $1.107,52$ & 132,90 \\
2014 & $1.129,01$ & 135,48 \\
2015 & $1.150,91$ & 138,11 \\
2016 & $1.173,24$ & 140,79 \\
2017 & $1.196,00$ & 143,52 \\
2018 & $1.219,20$ & 146,30 \\
2019 & $1.242,86$ & 149,14 \\
2020 & $1.266,97$ & 152,04 \\
2021 & $1.291,55$ & 154,99 \\
\hline
\end{tabular}

Elaboración propia. 


\subsection{Materias primas}

A continuación se analizarán las principales características de la materia prima que se va a utilizar en el proceso productivo de la mazamorra.

- Tocosh fresco.- El tocosh es un alimento que se consume desde épocas ancestrales en la sierra central de nuestro país, es el resultado de una tecnología andina de conservación de alimentos, en la cual se somete a la papa a un proceso de fermentación-putrefacción de manera artesanal; tiene un uso tanto medicinal como nutritivo en las zonas donde se elabora.

La preparación tradicional del tocosh consiste en utilizar como materia prima, papa seleccionada de la variedad «hualash»; esta es, según los productores, la mejor papa para preparar tocosh. Esta es introducida en pozas de dos o más metros de profundidad hechos al lado de un río o de alguna corriente de agua. Luego se procede a cubrir la papa con paja seca y piedras; posteriormente, el agua que corre a lado de la poza se filtrará y cubrirá todo su contenido. Para que el proceso convierta la papa en tocosh, esta deberá permanecer en la poza por un periodo aproximado de 5 a 6 meses. Durante los primeros meses es probable que se vea espuma en la poza como resultado del proceso de fermentación por el que está pasando la papa. $\mathrm{Al}$ cabo de un tiempo de iniciado el proceso, el tocosh estará listo para ser retirado de la poza y consumido.

El consumo tradicional es en forma de mazamorra, que se puede preparar a partir de tocosh fresco, recién sacado de la poza, o de harina de tocosh. También es frecuente consumirlo sancochado o incluso crudo.

En las fotos 1 y 2 se muestran imágenes captadas durante la realización de un trabajo de campo, en el cual se visitó el pueblo de Obas, ubicado en la provincia de Yarowilca, departamento de Huánuco, a 3.500 metros de altitud, donde se pudieron observar en forma directa las pozas de elaboración del tocosh, así como el proceso para sacarlo de las pozas y la manera tradicional de consumirlo. 
Foto 1

Extracción del tocosh

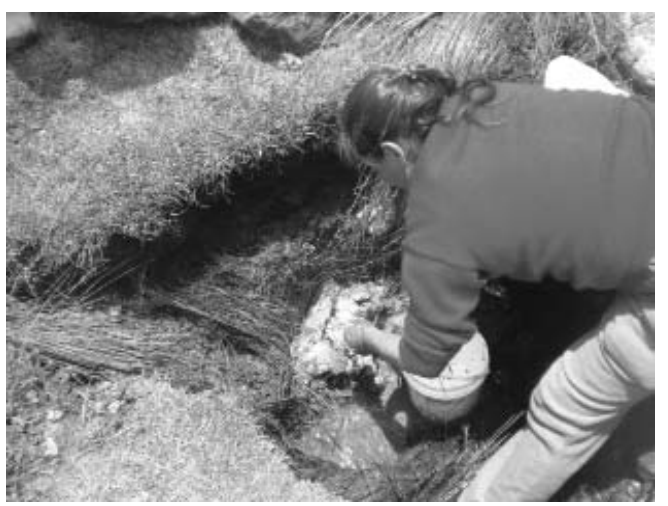

Foto 2

Familia campesina de Obas, Huánuco

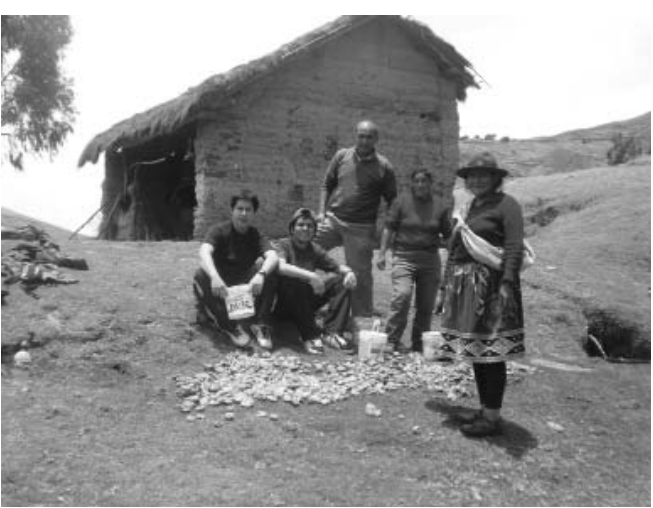

- Harina de maca.- La maca (Lepidium meyenii) es una planta herbácea, nativa de los Andes del Perú. Hay registros de que ha sido consumida desde épocas milenarias y de sus numerosas propiedades curativas y nutritivas. Ha sido procesada en gran escala durante los últimos años, por lo que existe una gran variedad de productos elaborados con maca, como harinas, néctares, cápsulas y mermeladas.

La principal zona de producción de la maca es la provincia de Junín, especialmente en las pampas de Junín, donde se procesa hasta la obtención de productos con valor agregado. La maca para el proyecto se comprará cuando se encuentre procesada en harina, la cual pasará a formar parte de la composición final del producto.

- Quinua lavada y perlada.- La quinua (Chenopodium quinoa) es un cereal oriundo de los Andes peruanos y bolivianos, del cual nuestro país es el segundo productor mundial. Fue uno de los principales componentes de la dieta alimenticia de las sociedades preincaicas e incaicas.

En el Perú existen muchas industrias que procesan la quinua, destinando sus productos tanto a la exportación como al mercado interno. Se presenta en forma de harina o como grano perlado y lavado, esto último es una operación necesaria, ya que la quinua originalmente tiene un sabor bastante amargo, debido a la presencia de la saponina. 
Puede crecer a cualquier altitud, pero se desarrolla por encima de los 2.500 metros de altitud, por ello es posible encontrar este producto en zonas cercanas a donde se produce el tocosh; los principales productores y procesadores se encuentran en el sur del país.

- Leche en polvo.- Es la leche pasteurizada, deshidratada y muy rica en calcio; este tipo de presentación facilita en gran medida la manipulación y el uso del lácteo, al darle condiciones más simples de almacenamiento.

La mayor cantidad de leche en polvo que se comercializa en el país es importada, y muchos proveedores la venden al por mayor. Se incluye determinada cantidad de leche dentro de la formulación de la mazamorra por su alto grado alimenticio y porque al interactuar con el tocosh ayuda a disminuir, en cierto grado, el olor de este.

- Azúcar.- Es la sacarosa obtenida generalmente de la caña de azúcar; es un ingrediente muy importante y de gran proporción para nuestro producto propuesto, ya que al ser este introducido como un postre, se necesita que sea agradable al gusto, lo que en gran medida se logra con el dulzor del azúcar.

El Perú figura entre los grandes productores mundiales de este insumo, y existe gran disponibilidad para poder abastecerse de él.

- Fécula de papa.- Es el almidón obtenido mediante un proceso en el que se trituran las papas para liberar los granos de almidón de las células destruidas, estos se lavan y se secan para obtener el almidón en polvo.

Este insumo se utiliza en nuestro producto principalmente para ayudar en su capacidad de espesar. Si bien la harina de tocosh es de por sí un espesante, una cantidad de fécula de papa ayudará a que el producto rinda más porciones.

Aunque el Perú es un país productor de papa, la industria de fabricación de fécula a partir de este tubérculo es muy baja y muy poco competitiva; por ello este insumo es importado de los países de mayor producción de papa en el mundo, como Holanda.

\subsection{Disponibilidad de insumos}

Se considera como área de influencia del proyecto la zona central del Perú, comprendida por los departamentos de Lima, Ancash, Pasco, Huánuco y Junín. 
En estos departamentos es donde abundan las materias primas necesarias para la elaboración del producto propuesto en el presente proyecto. Por ejemplo, el tocosh, la materia prima principal, es producido en mayor cantidad en la sierra de Huánuco; sin embargo, su elaboración es bastante artesanal y toma bastante tiempo obtener la cantidad de tocosh requerida, por lo que la disponibilidad de este insumo va a ser muy limitada.

Para tener una visión real de la disponibilidad de tocosh, y debido a la falta de información sobre este producto, se realizó un trabajo de campo en Huánuco (foto 3), donde se visitaron los principales puntos de venta de tocosh. Se encontró que es un mercado de difícil acceso; la mayoría de comerciantes de este insumo son minoristas, y son abastecidos semanalmente por algún acopiador. También se visitó el campo ferial Puelles, donde los días sábado en la madrugada, se realiza la venta al por mayor de diversos productos agrícolas; en este campo ferial se encuentran unos pocos vendedores de tocosh, quienes semanalmente llevan camionetas cargados con baldes llenos de tocosh; pero que la mayoría de esos baldes ya están vendidos; si se desea comprar grandes cantidades hay que pedirlas con anticipación. La venta se realiza al peso y se hace de manera bastante rudimentaria. A partir de la conversación directa con los vendedores se pudo estimar que es posible abastecerse semanalmente de un promedio de 2.000 kilogramos; sin embargo, también se pudo concluir que será necesario realizar un trabajo

Foto 3

Mercado minorista de tocosh, Huánuco

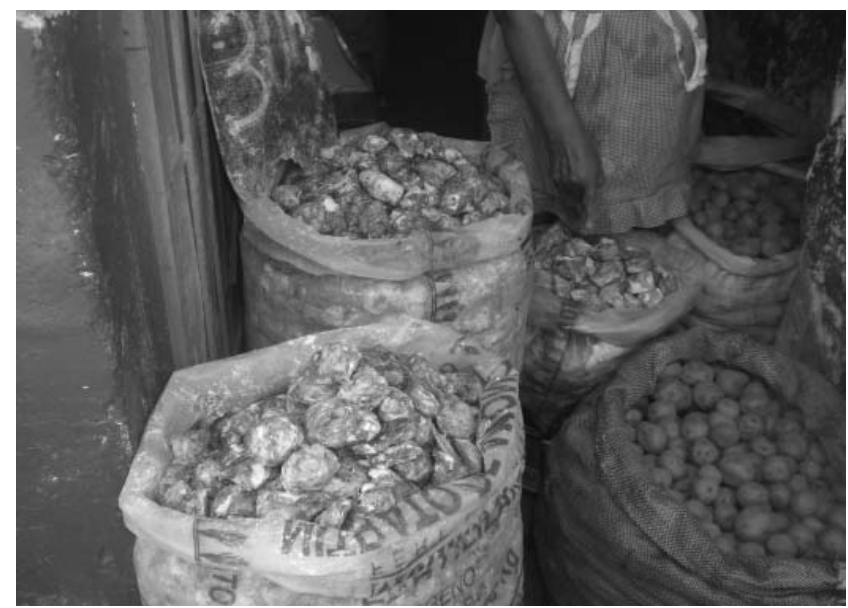


directo con los proveedores para lograr un abastecimiento constante, tanto en cantidad como en calidad.

En lo que respecta a los otros insumos, la mejor harina de maca y en mayor cantidad se puede encontrar en el departamento de Junín, donde existe en grandes cantidades y su disponibilidad no es limitante. La quinua, como se mencionó, se produce en gran cantidad en el Perú y es bastante usual encontrarla en la forma en la que será adquirida para el proyecto. La leche en polvo y la fécula de papa son productos principalmente importados, ya que la producción peruana es mínima, el primero generalmente de Uruguay, Argentina y Nueva Zelanda; el segundo de Alemania y Holanda; en ambos casos hay un buen número de importadores en Lima que pueden abastecer de grandes volúmenes de manera permanente. Finalmente, el azúcar es producida en el norte de nuestro país, y es posible abastecerse de grandes cantidades mediante distribuidores y comerciantes mayoristas en todas las principales ciudades del Perú.

\section{LOCALIZACIÓN DE LA PLANTA}

Para determinar la óptima localización geográfica de la planta productora se tuvieron en cuenta factores determinantes como la proximidad a las materias primas, la cercanía al mercado, los requerimientos de infraestructura industrial y las condiciones socioeconómicas. De acuerdo con estos parámetros, se establecieron como posibles ubicaciones las regiones Lima, Ancash y Huánuco.

Con el fin de establecer la mejor macrolocalización entre estas regiones se aplicó el método de ranking de factores, teniendo en cuenta, además de los antes mencionados, las condiciones climáticas, el abastecimiento de agua, y otros. Producto de la evaluación resultó ganadora la región Huánuco, lugar donde se ubicaría la planta.

En cuanto a la microlocalización se consideraron las provincias de Ambo, Huánuco y Dos de Mayo; la primera por ser la puerta de ingreso a la región Huánuco con dirección a Lima, la segunda por ser la capital de la región y albergar el mayor desarrollo económico de la zona, y la última por ser la provincia más desarrollada de la sierra huanuqueña y estar muy cerca de las zonas de mayor producción de tocosh.

Aplicando nuevamente el método de ranking de factores, se obtuvo como ganadora la provincia de Huánuco, principalmente por el mayor 
desarrollo económico y de infraestructura que presenta, así como por las mejores vías de acceso y cercanía a la materia prima principal que es el tocosh. Específicamente la planta se podría ubicar en el distrito de Pillko Marca, zona que ofrece las mejores condiciones para la implementación del proyecto.

\section{Figura 1}

Localización de la planta
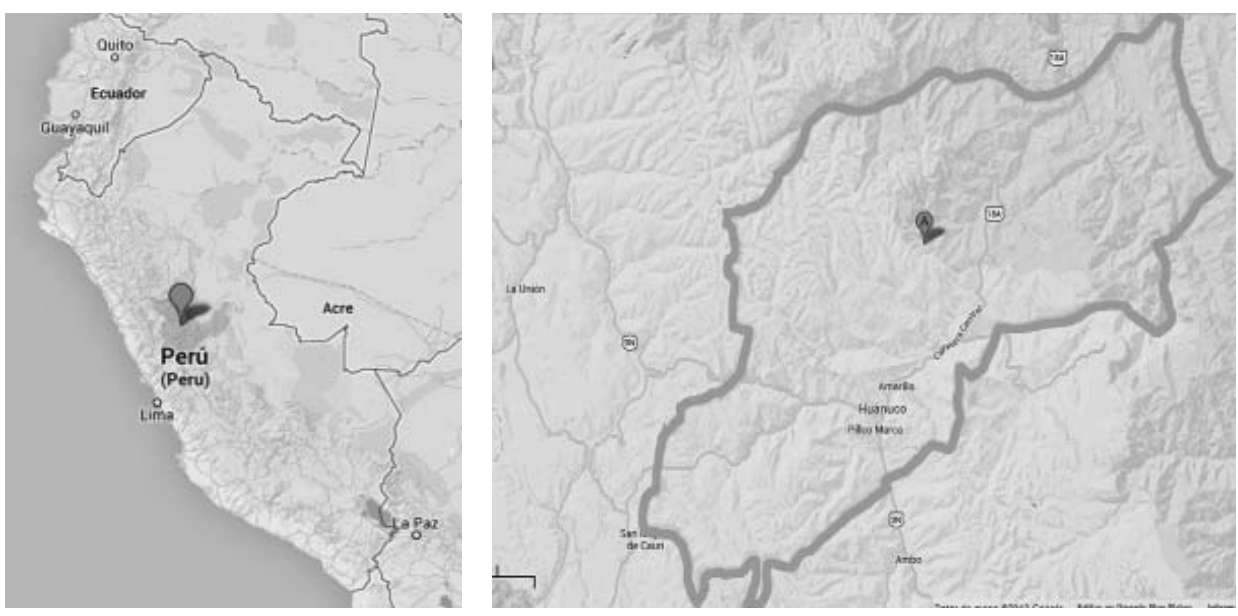

Fuente: Google maps.

\section{TAMAÑO DE LA PLANTA}

Para determinar el tamaño adecuado de la planta se procedió a establecer las relaciones de tamaño máximo, mínimo y limitante, como se muestran a continuación: 
Tabla 5

Resumen de relaciones

\begin{tabular}{lc}
\hline Tipo de relación & $\begin{array}{c}\text { Tamaño de planta } \\
\text { (t/año) }\end{array}$ \\
\hline Mercado & 162,00 \\
Recursos productivos & 100,63 \\
Tecnología & 139,47 \\
Punto de equilibrio & 40,86 \\
\hline
\end{tabular}

Elaboración propia.

Debido a las limitaciones de abastecimiento de recursos productivos, en específico de tocosh, el tamaño seleccionado como óptimo para la planta es de 100,63 toneladas por año.

\section{INGENIERÍA DEL PROYECTO}

\subsection{Especificaciones técnicas del producto}

La mazamorra resulta de la mezcla de harinas de tocosh, maca, quinua, a la que se agregan leche en polvo, fécula de papa y azúcar, que al ser combinado con agua y añadirse calor, luego de una cocción rápida, de aproximadamente cinco minutos, se constituirá en un producto de consistencia semisólida típica de los postres llamados mazamorras.

Tabla 6

Especificaciones del producto

\begin{tabular}{ll}
\hline Característica & Especificación \\
\hline Peso neto & 200 gramos \\
Color & Marrón claro homogéneo \\
Olor & Característico \\
Sabor & Característico, dulce \\
Vida útil & 12 meses \\
Empaque & Bolsas de polietileno \\
Embalaje & Bolsones de 24 unidades \\
\hline
\end{tabular}

Elaboración propia. 


\subsection{Descripción del proceso de producción}

El proceso productivo consta de nueve etapas que se explican a continuación:

- Lavado.- El tocosh fresco será llevado desde el almacén de materia prima hasta los lavaderos, donde mediante el uso de duchas de aspersión de agua se procederá a realizar el lavado unidad por unidad; en este proceso se elimina la tierra y otros residuos que están adheridos a la cáscara del tocosh.

- Oreado.- Una vez lavado, el tocosh pasará en jabas por un proceso de oreado hasta el día siguiente; en este punto, mediante el uso de aire se busca reducir la humedad externa del tocosh para hacer más fácil el pelado del producto y disminuir la merma al momento de quitarle la cáscara.

- Pelado y selección.- Después de haber sido oreadas, las jabas con tocosh serán llevadas al área de pelado donde, sobre unas mesas de acero, se procederá a quitarle la cáscara; este proceso se hará manualmente, uno por uno. Al mismo tiempo que se realiza el pelado, el operario irá retirando el producto que se encuentre en mal estado.

- Cortado.- Para optimizar la deshidratación del tocosh, luego de pelado, se procederá a cortarlo con el fin de aumentar la superficie de contacto con el aire caliente en la deshidratadora y optimizar el consumo de energía. Este proceso se realizará mediante una picadora manual, dentro de la cual se introducirán las bandejas con el tocosh y el operario accionará manualmente una guillotina que cortará el producto.

- Oreado.- El segundo oreado también durará toda la noche; se realizará después del cortado y antes de que entre a la máquina deshidratadora; para ello habrá un área especial ventilada donde se depositarán las bandejas provenientes del cortado en estantes de metal. Aquí se busca reducir la humedad del tocosh y optimizar aún más el proceso de deshidratación. 
- Deshidratación.- Se realizará en una deshidratadora de lecho fluidizado, máquina a la que entrará el tocosh después del segundo oreado a una humedad aproximada de $45 \%$. En este proceso se deberá verificar constantemente la temperatura del aire caliente (alrededor de 60 grados centígrados). Una vez obtenido el tocosh deshidratado con una humedad aproximada de $8 \%$ se almacenará en costales para pasar al siguiente proceso.

- Molienda.- Una vez deshidratado, el tocosh estará listo para ser convertido en harina; esta operación se llevará a cabo en el molino de martillos, donde se obtendrá harina de tocosh fina con una medida de malla de 0,5 milímetros.

- Mezclado.- Cuando la harina de tocosh esté lista se podrá mezclar con los otros insumos, que son la harina de maca, el azúcar, la fécula de papa, la leche en polvo y la harina de quinua, la cual se obtuvo de la molienda de la quinua lavada, de acuerdo a la formulación del producto final; para esta mezcla se realizará una dosificación previa de cada uno de los componentes, de acuerdo a los batch de mezclado de la máquina.

- Envasado.- Finalmente, una vez obtenido el producto mezclado y homogéneo se procederá al envasado en una máquina automática, la cual dosificará, envasará y sellará las bolsas, obteniéndose así la mezcla lista para preparar mazamorra como producto terminado.

\subsection{Diagrama de operaciones}

A continuación se muestra el diagrama de operaciones para la producción de mazamorra de tocosh con maca, quinua, fécula de papa, azúcar y leche. 
Diagrama 1

DOP

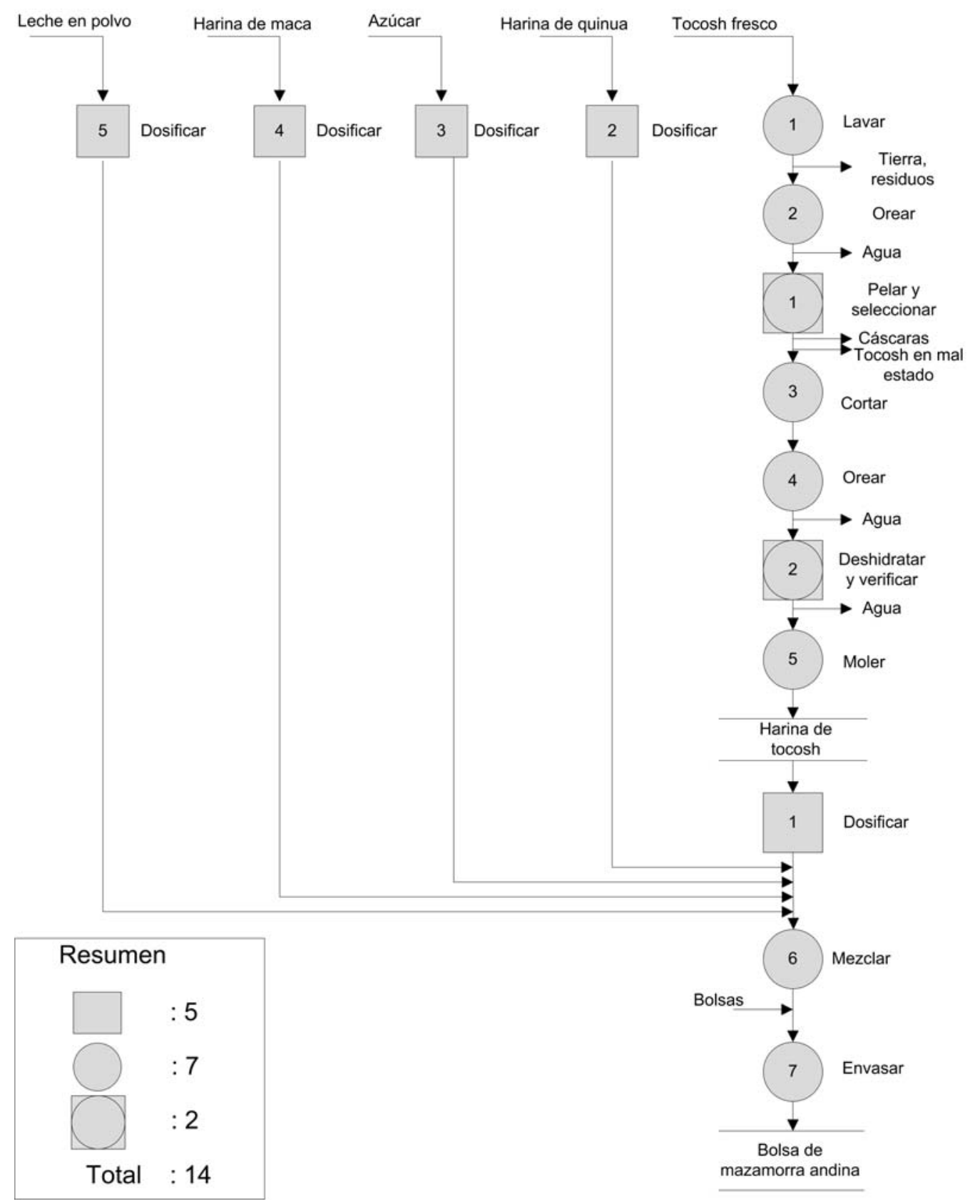

Elaboración propia. 


\subsection{Capacidad instalada}

Para determinar la cantidad de máquinas se tomó en cuenta el tamaño de planta elegido anteriormente, luego para hallar la capacidad instalada se estimaron las capacidades y los tiempos estándares de cada una de las operaciones, tanto de las manuales como de las que precisan la utilización de maquinaria. Esta estimación se realizó mediante diferentes pruebas de laboratorio y una corrida de producción en una planta piloto ubicada en la ciudad de Huánuco a la que se tuvo acceso.

Foto 4

Pruebas de laboratorio

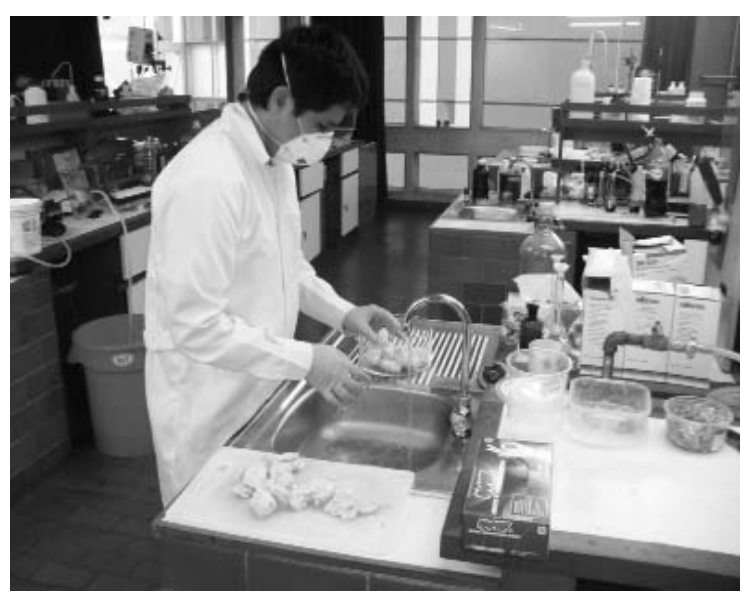

Foto 5

Producción en planta piloto

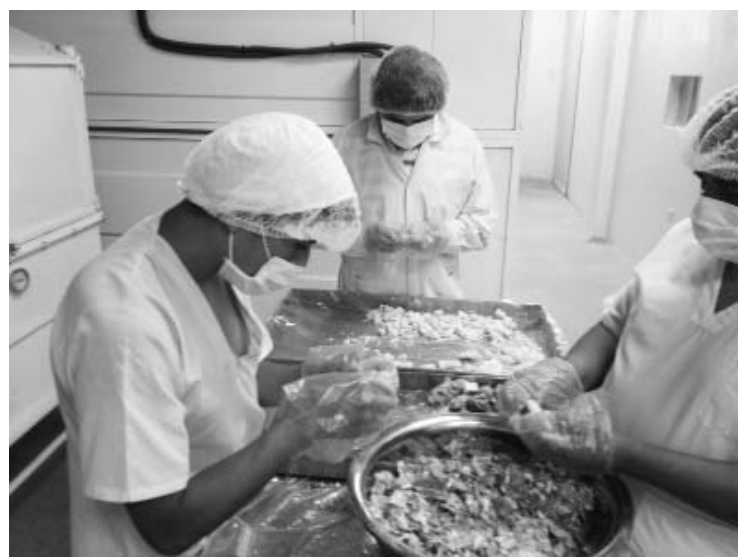




\subsection{Resguardo de la calidad}

El resguardo de la calidad será guiado a partir de la puesta en práctica de un manual de buenas prácticas de manufactura (BPM), un plan de higiene y saneamiento, y la aplicación del sistema HACCP.

Habrá un control de calidad tanto de las materias primas como del proceso y del producto terminado; así, todo lote de producción tendrá que pasar por un análisis fisicoquímico y microbiológico para certificar que está apto para su comercialización.

Una vez realizados los pasos exigidos para la aplicación del sistema HACCP, que incluye el análisis y la evaluación de los peligros, y la selección de los puntos de control y vigilancia de estos puntos, se estableció como punto crítico de control (PCC) para nuestro proceso la operación de pelado y selección, ya que si esta no se realiza bien existe la posibilidad de que algunos peligros químicos y microbiológicos lleguen hasta el producto final. A continuación se muestran las fotos de algunos tocosh frescos que deben ser retirados en esta operación.

\subsection{Disposición de planta}

Para proponer la disposición de la planta productora de mazamorra se analizaron los diferentes factores que influyen en el diseño, como el edificio, el servicio, la espera, entre otros. Para las zonas productivas se aplicó el método de análisis de Guerchet, en el cual se establecieron las superficies estáticas, de gravitación y de evolución por cada zona productiva. Una vez definidos todos los factores se propusieron los requerimientos mínimos de espacio de cada una de las áreas que conformarán la planta.

Finalmente, con la aplicación de un análisis de relaciones se distribuyeron las distintas áreas con sus respectivas dimensiones, llegándose a un plano de distribución al detalle. A continuación se muestra dicho plano indicándose cada área y sus dimensiones. 
Figura 2

Disposición en detalle

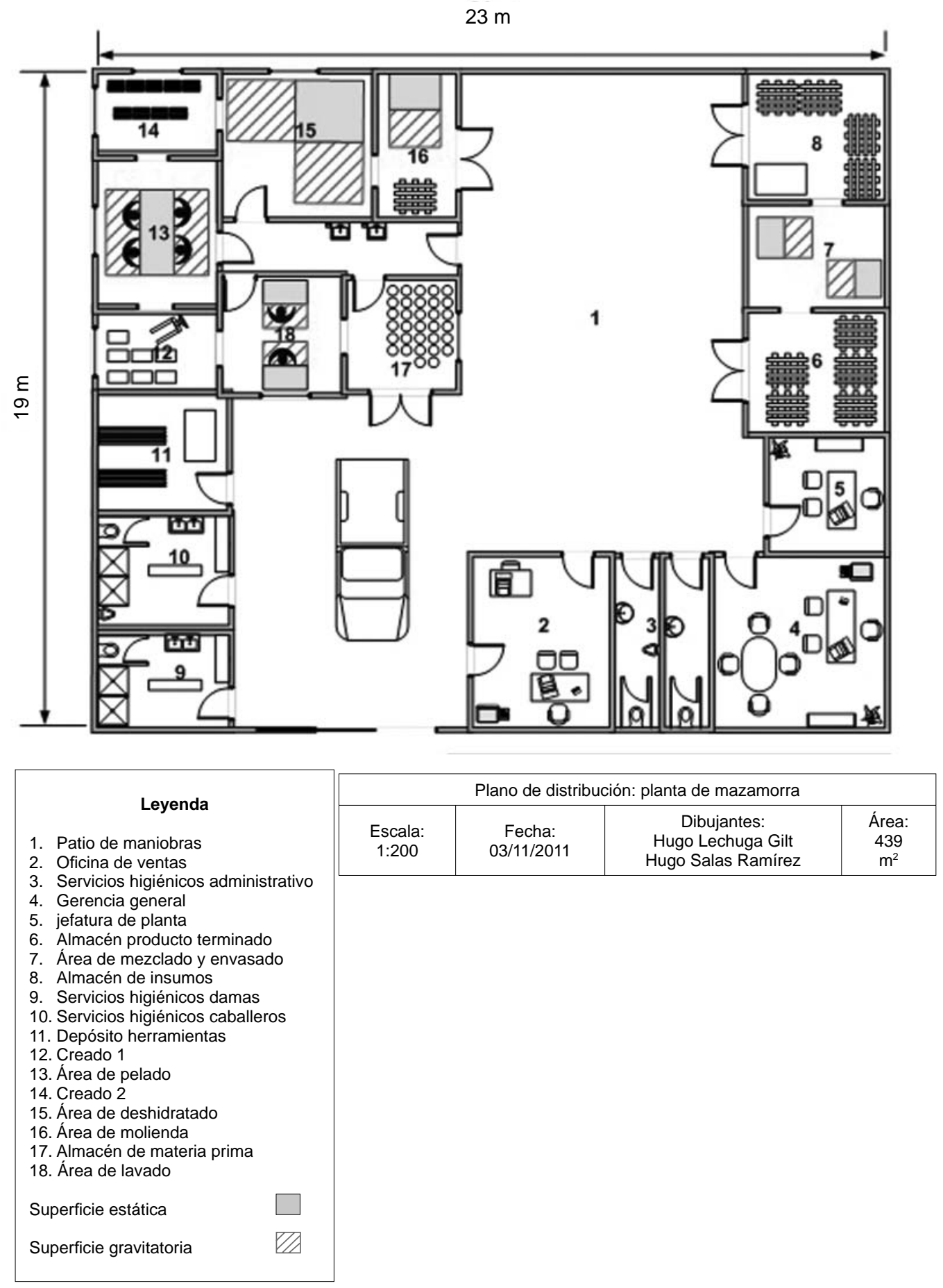




\section{ASPECTOS ECONÓMICOS Y FINANCIEROS}

\subsection{Inversiones}

En esta parte de la investigación se realizó una estimación del capital total necesario para la construcción, implementación y puesta en marcha del proyecto.

A partir de la inversión total para máquinas y equipos, la cual asciende a $\mathrm{S} / .122 .774$, se pudo hacer la estimación de los demás rubros de activos tangibles e intangibles, mediante la aplicación de la técnica de estimación de inversiones de Lang.

Tabla 7

Estimado de la inversión total

\begin{tabular}{lr}
\hline Activos fijos tangibles & Monto (S/.) \\
\hline Equipo comprado, despachado & $122.774,00$ \\
Instalación del equipo & $55.248,30$ \\
Instrumentación y control & $11.049,66$ \\
Tuberías & $19.643,84$ \\
Eléctricos & $12.277,40$ \\
Edificios & $304.560,00$ \\
Mejoras de terreno & $12.420,00$ \\
Servicios & $49.109,60$ \\
Terreno & $62.100,00$ \\
Total activo fijo tangible & $649.182,80$ \\
\hline Activos fijos intangibles & \\
\hline Ingeniería y supervisión & $40.515,42$ \\
Gastos de construcción & $47.881,86$ \\
Contratistas & $20.871,58$ \\
Contingencias & $41.743,16$ \\
Total activos fijos intangibles & $151.012,02$ \\
Total activos fijos & $\mathbf{8 0 0 . 1 9 4 , 8 2}$ \\
Capital de trabajo & $\mathbf{8 0 . 0 1 9 , 4 8}$ \\
Inversión total & $\mathbf{8 8 0 . 2 1 4 , 3 0}$ \\
\hline
\end{tabular}

Elaboración propia. 


\subsection{Financiamiento}

Parte de la inversión será financiada por alguna entidad que otorgue préstamos. Para el caso de esta investigación tomaremos como referencia la Corporación Financiera de Desarrollo (Cofide), entidad que proporciona recursos para la realización de proyectos, con un $16 \%$ de tasa de interés, que es la tasa que en promedio maneja esta institución.

A continuación se muestra un cuadro donde se señala la repartición de la inversión total entre recursos financiados y recursos propios.

Tabla 8

Partición de la inversión

\begin{tabular}{lll}
\hline Fuente & $\%$ & Monto (SI.) \\
\hline Financiado & 65 & $572.139,30$ \\
Propio & 35 & $308.075,01$ \\
\hline
\end{tabular}

Elaboración propia.

\subsection{Evaluación económica y financiera}

Se realizó una evaluación económica, en la cual se asume que el total de la inversión será cubierta con recursos propios; y una evaluación financiera, en la cual los recursos para la inversión fueron repartidos entre propios y financiados, como se mencionó anteriormente.

Aplicando un costo de capital de $20 \%$, se obtuvieron los siguientes resultados para cada uno de los casos.

Tabla 9

Evaluación económica y financiera

\begin{tabular}{llc}
\hline Económica & Valor actual neto & S/. 148.285,62 \\
\hline Económica & Tasa interna de retorno & $24,01 \%$ \\
Económica & Beneficio/costo & 1,17 \\
Económica & Periodo de recupero & Antes del 8. ${ }^{\circ}$ año \\
Financiera & Valor actual neto & S/. 289.586,14 \\
Financiera & Tasa interna de retorno & $36,28 \%$ \\
Financiera & Beneficio/costo & 2,20 \\
Financiera & Periodo de recupero & Antes del 6. ${ }^{\circ}$ año \\
\hline
\end{tabular}

Elaboración propia. 
Para ambos casos todos los indicadores de la evaluación económica y financiera resultaron positivos y aceptables, lo cual indica que el proyecto sí es viable en cuanto a estos aspectos.

\subsection{Análisis de sensibilidad}

Se puede concluir que el proyecto es significativamente sensible a la variación de la demanda, por lo cual se deberá tener planes de contingencia en caso de que ocurra un escenario pesimista; por otro lado, el proyecto no es significativamente sensible a la variación del precio del tocosh fresco; sin embargo, es una variable que se debería tener vigilada constantemente.

Tabla 10

Escenarios según la variación de la demanda y costo del tocosh

\begin{tabular}{lcrrr}
\hline Variable & Escenario & \multicolumn{1}{c}{$\mathbf{+ 1 0 \%}$} & Sin variación & \multicolumn{1}{c}{$\mathbf{- 1 0 \%}$} \\
\hline Demanda & VAN económico & $512.812,47$ & $148.285,62$ & $-216.241,23$ \\
Demanda & TIR económico & $33 \%$ & $24 \%$ & $13,6 \%$ \\
Demanda & VAN financiero & $654.112,99$ & $289.586,14$ & $-74.940,71$ \\
Demanda & TIR financiero & $56 \%$ & $36 \%$ & $16 \%$ \\
Costo del tocosh & VAN económico & $56.516,62$ & $148.285,62$ & $240.054,62$ \\
Costo del tocosh & TIR económico & $22 \%$ & $24 \%$ & $26,39 \%$ \\
Costo del tocosh & VAN financiero & $197.817,14$ & $289.586,14$ & $381.355,13$ \\
Costo del tocosh & TIR financiero & $31,18 \%$ & $36 \%$ & $41,35 \%$ \\
\hline
\end{tabular}

Elaboración propia.

\section{Evaluación social del proyecto}

Para realizar una correcta identificación de las zonas en las que influirá el proyecto las hemos dividido en tres, según cuánto sean impactadas por la realización del proyecto; así, hemos identificado una zona crítica, otra de influencia directa y finalmente una de influencia indirecta. En la matriz que se presenta en la tabla 11, se detallan las principales acciones tomadas sobre los impactos identificados en la instalación de la planta. 


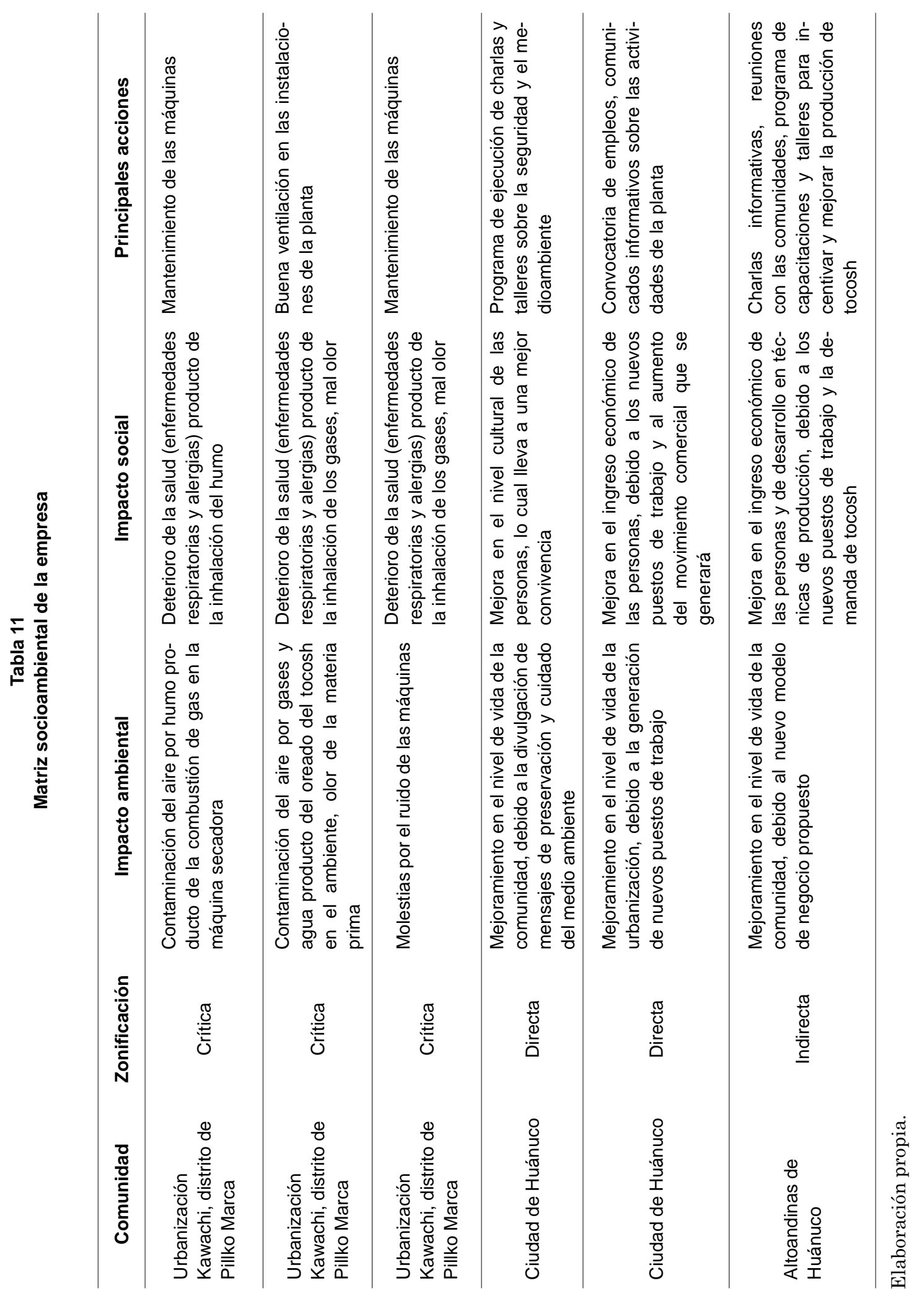




\section{CONCLUSIONES}

- En la presente investigación se ha constatado que la instalación de una planta productora de mazamorra de tocosh con maca, quinua, azúcar, fécula de papa y leche es factible, en términos del estudio de prefactibilidad que se realizó.

- El proyecto es viable con respecto al mercado, debido a que existe una demanda potencial de 136,42 toneladas para el proyecto en el año 2013 y está en crecimiento.

- El proyecto es viable económica y financieramente, ya que después de realizar un exhaustivo análisis de la inversión que acarrearía, de todos los costos de producción y de los gastos administrativos y de considerarlos durante toda la vida útil del proyecto, se obtuvieron resultados positivos y por encima de las expectativas; el análisis económico arrojó como principales indicadores de resultados un VAN de S/. 148.285,62 y un TIR de 24,01\%, mientras que los resultados financieros fueron un VAN de S/. 289.586,14 y un TIR de 36,28\%.

- El proyecto tendrá un alto impacto positivo dentro de las áreas de influencia, ya que dará un mayor movimiento industrial a una zona que se caracteriza por operaciones económicas comerciales y no de transformación. También habrá un fuerte impacto dentro de las comunidades productoras de tocosh, ya que podrán encontrar un cliente fijo para sus producciones y podrán, poco a poco, ir tecnificando y mejorando sus productos y calidad de vida.

\section{RECOMENDACIONES}

- Realizar un estudio de mercado de mayor detalle, con la aplicación de un trabajo de campo con validez estadística, que permita tener un resultado validado de que el producto será aceptado por el mercado objetivo.

- Investigar qué otros productos se pueden fabricar a partir del tocosh industrializado, ya que la harina que se obtiene para elaborar la mazamorra podría servir también para otros artículos.

- Trabajar en cooperación con los proveedores y productores de tocosh desde un principio, con el fin de que se puedan lograr mejores lazos de confianza, los que en la actualidad no existen por lo cual resulta un mercado de abastecimiento muy informal, y con ello mejorar la calidad del producto y potenciar su producción. 
- Estudiar la factibilidad de tecnificar la producción de tocosh, que es muy artesanal, para verificar la posibilidad de integración hacia atrás del proyecto. Es decir, que se cuente con áreas propias de producción de tocosh en la sierra de Huánuco.

\section{REFERENCIAS}

Asociación Peruana de Empresas de Investigación de Mercados (Apeim) (2011). «Niveles socioeconómicos 2011, total Perú y Lima metropolitana». Fórmula Apeim - NSE. Recuperado el 17 de agosto del 2011, de http://www.apeim.com.pe/niveles.html

Colina, M. L. (2010). Deshidratación de alimentos (1 ${ }^{\mathrm{a}}$ ed.). México D. F.: Trillas.

Huerta, E., \& Tarazona, J. (2011). Situación económica, social y de las inversiones de Ancash. Ancash: Universidad Santiago Antúnez de Mayolo.

Instituto Nacional de Estadística e Informática - INEI (2011). Población total, tasa de crecimiento anual. Recuperado el 2 de setiembre del 2011, de http://www.inei.gob.pe/perucifrasHTM/inf-dem/ cuadro.. sp $?$ cod $=3643 \&$ name $=$ po02\&ext $=$ gif.

Ipsos Apoyo (2011). Perfil del ama de casa 2010. Informe gerencial de marketing. Lima: Ipsos Apoyo.

Mallqui, J. (2001). Perfil técnico y de comercialización del tocosh. Huánuco: Gobierno Regional de Huánuco.

Ministerio de Salud (2009). Plan multianual de inversiones 2009-2013. Recuperado el 9 de setiembre del 2011, de http://www.minsa.gob. pe/diresahuanuco/COIN/plan20092013.pdf

Ministerio de Salud (2010). Habilitación sanitaria. Recuperado el 10 de setiembre de 2011, de http://www.digesa.minsa.gob.pe/DHAZ/ habilitacion.asp

Perú (2006). Resolución Ministerial 449-2006/MINSA. Norma sanitaria para la aplicación del sistema HACCP en la fabricación de alimentos y bebidas. Lima. 
Hugo Lechuga Gilt, Hugo Ilan Salas Ramírez

Perú (1998). Decreto Supremo 007-98-SA. Reglamento sobre Vigilancia y Control Sanitario de Alimentos y Bebidas. Lima.

Tagle, G. (2010). Estudio preliminar para la instalación de una planta procesadora de mazamorra de oca en polvo. Seminario de Investigación de la Facultad de Ingeniería Industrial. Lima: Universidad de Lima. 